\title{
A New Rapid and Cost-Effective Method for Detection of Phages, ICEs and Virulence Factors Encoded by Streptococcus pyogenes
}

\author{
ANNA L. BOREK ${ }^{1}$, JOANNA WILEMSKA ${ }^{1,3}$, RADOSŁAW IZDEBSKI², WALERIA HRYNIEWICZ ${ }^{1}$ \\ and IZABELA SITKIEWICZ ${ }^{1 *}$
}

\author{
${ }^{1}$ Department of Epidemiology and Clinical Microbiology, National Medicines Institute, Warsaw, Poland \\ ${ }^{2}$ Department of Molecular Microbiology, National Medicines Institute, Warsaw, Poland \\ ${ }^{3}$ Current address: Department of Clinical Cytology, The Medical Centre of Postgraduate Education, Warsaw, Poland
}

Received 27 June 2011, revised 12 July 2011, accepted 14 July 2011

\begin{abstract}
Streptococcus pyogenes (group A Streptococcus, GAS) is a human pathogen that causes diseases of various intensity, from mild strep throat to life threatening invasive infections and postinfectional sequelae. S. pyogenes encodes multiple, often phage encoded, virulence factors and their presence is related to severity of the disease. Acquisition of mobile genetic elements, carrying virulence factors, as phages or ICEs (integrative and cojugative elements) has been shown previously to promote selection of virulent clones. We designed the system of eight low volume multi- and one singleplex PCR reactions to detect genes encoding twenty virulence factors ( $s p d 3, s d c, s d a B, s d a D, s p e B, s p y C E P$, scpA, mac, sic, speL, $K, M, C, I, A, H, G$, J, smeZ and ssa) and twenty one phage and ICE integration sites described so far for $S$. pyogenes. Classification of strains based on the phage and virulence factors absence or presence, correlates with PFGE MLST and emm typing results. We developed a novel, fast and cost effective system that can be used to detect GAS virulence factors. Moreover, this system may become an alternative and effective system to differentiate between GAS strains.
\end{abstract}

Ke y word s: Streptococcus pyogenes, GAS, superantigens, virulence factors, typing, phage

\section{Introduction}

Streptococcus pyogenes (group A Streptococcus, GAS) is an important human pathogen that causes a broad spectrum of skin and mucosal surface infections. GAS diseases range from mild, such as streptococcal pharyngitis or impetigo, to severe toxin-mediated, among which are necrotizing fasciitis or toxic shock syndrome and postinfectious diseases (Cunningham, 2000). GAS is responsible for over 600 millions of new infections every year and causes half a million deaths as a result of infections and post-infectional sequelae (Carapetis et al., 2005).

The success of GAS as a pathogen relies on the production of multiple virulence factors involved in various aspects of host-pathogen interactions (Tart et al., 2007). Initial contact between bacteria and the host is achieved by the activity of multiple adhesins produced by GAS which bind host proteins and extracellular matrix proteins such as plasminogen, collagen, keratin (for a review see Courtney et al., 2002; Cunningham, 2000; Oehmcke et al., 2010; Smeesters et al., 2010).
After initial contact, bacteria invade host tissues and often disseminate causing systemic reaction (Tart et al., 2007). Multiple classes of GAS virulence factors such as proteases, DNases and pyrogenic toxins (superantigens) are involved in interaction between bacteria and the host in post attachment phase.

Major surface adhesin - M protein, is involved in tissue invasion and interaction with human immune system (Perez-Caballero et al., 2004). Other elements of the human immune system are inactivated by set of specialized proteases. ScpA a highly specific peptidase encoded by $\operatorname{scp} A$ gene degrades C5a factor of the complement (Cleary et al., 1992). SpeB is a cysteine protease that can inactivate $\mathrm{C} 3 \mathrm{~b}$ factor of the complement (Terao et al., 2008) and multiple other host factors involved in immune response such as interleukin- $1 \mathrm{~b}$ precursor and immunoglobulins (Chiang-Ni and $\mathrm{Wu}, 2008)$. In addition SpeB is involved in tissue destruction by activation of pro-matrix metallo-proteases and degradation of fibronectin, vitronectin, plasminogen and kininogen (Chiang-Ni and $\mathrm{Wu}, 2008$ ). The protease MAC/IdeS cleaves specifically human IgG

\footnotetext{
* Corresponding author: I. Sitkiewicz, Department of Epidemiology and Clinical Microbiology, National Medicines Institute,
} Chełmska 30/34, 00-725 Warszawa, Poland; phone (+48 22) 841 12 22; fax (+48 22) 84129 41; e-mail: isitkiewicz@cls.edu.pl 
(von Pawel-Rammingen et al., 2002). Recently discovered protease SpyCEP is involved in degradation of chemokines and chemotactic factors as interleukin-8, granulocyte chemotactic protein-2, growth related oncogene $\alpha$ and $\beta$ and macrophage inflammatory protein 2-a (Edwards et al., 2005; Kurupati et al., 2010; Sumby et al., 2008; Zinkernagel et al., 2008).

DNases produced by GAS are involved in dissemination of bacteria and escape from neutrophil extracellular traps (Sumby et al., 2005; Walker et al., 2007). And finally, large group of toxins encoded by GAS genes (spel, speK, speM, speC, speI, speA, speH, speG, speJ, sme $Z$ and $s s a$ ) is involved in systemic toxicity.

Some of the GAS virulence factors, e.g. SpeB, ScpA, SpyCEP, Mac, SdaB and SpeG, are chromosomally encoded, however, large fraction of virulence factors such as majority of DNAses and superantigens e.g. SpeA, SpeC, SpeH and SSA are encoded by mobile genetic elements - phages and conjugative mobile elements integrated into the chromosome (ICEs - integrative and cojugative elements) (Beres and Musser, 2007). Based on the comparison of genome sequences of multiple GAS strains, metagenome of GAS contains on average about $10 \%$ of exogenous elements (Beres and Musser, 2007; Ferretti et al., 2001). So far, 67 mobile elements (55 prophages and 12 ICE elements) integrated at 21 distinct loci of the core chromosome in the 12 GAS genomes have been identified (Beres and Musser, 2007) (Table I).

Over the years, multiple serological, restriction fragment based and PCR based methods of GAS typing and virulence factors detection were used (Cleary et al., 1988; Commons et al., 2008; Hartas et al., 1998; Koller et al., 2010; Lintges et al., 2007; Matsumoto et al., 2003; Maxted et al., 1973; Moody et al., 1965; Nandi et al., 2008; Schmitz et al., 2003; Seppala et al., 1994; Swift et al., 1943). Each of the methods presents various advantages and disadvantages. Serological assays are usually less precise than molecular methods. Methods based on the analysis of restriction patterns, and methods based on random amplification, are often difficult for analysis and comparison. Multiple PCR assays utilizing specific targets were developed before the era of massive genome sequencing that allows including the knowledge of allelic variations between strains of various serotypes in the design of more specific systems. Also, multiple PCR based systems were designed mostly as singleplex reactions what increases screening costs in case of detection of multiple virulence factors.

Currently, to determine the relationships between GAS isolates and strains, three major methods are typically used. The first method, which is regarded as a golden standard by many laboratories, is pulsed field gel electrophoresis (PFGE) typing (Bert et al., 1997). PFGE is often recommended as a reference method in outbreak investigations, especially for food borne diseases (http://www.cdc.gov/pulsenet/, http://www. medvetnet.org $/ \mathrm{cms} /$ ). PFGE is a method based on restriction fragment size polymorphism. Chromosomal DNA is released from bacteria and digested with rare cutting restriction enzyme directly in agarose gel and fragments are separated using alternating voltage gradient (for a review see (Herschleb et al., 2007; Slater, 2009)). PFGE typing detects rather large and recent evolutionary changes in bacterial DNA such as insertion or excision of a phage, large insertions and deletions and mutations resulting in a loss or appearance of a new restriction site (Tenover et al., 1995). Two strains are related to each other when the number of differences in restriction patterns is below 7 (Tenover et al., 1995). PFGE is a technique with high discriminatory power, but it is time consuming and available protocols require from minimum two days to over one week to determine PFGE type of the strain (Herschleb et al., 2007). What's equally important, the technique requires relatively expensive equipment, skilled labor and the results of PFGE are often difficult to compare between laboratories.

The second method used routinely in GAS epidemiology is emm typing. Emm typing, is a molecular equivalent of serotyping and allows grouping of GAS strains into serotypes/genotypes based on the type of the surface M protein (Facklam et al., 1999; Hoe et al., 1999). It is an easy and straightforward method that utilizes sequencing of a portion of the emm gene, which encodes hypervariable region of the $\mathrm{M}$ protein. The major advantage of molecular emm typing is rapid identification of novel variants of $\mathrm{M}$ protein responsible for new serotypes. The emm typing requires PCR amplification, purification of the amplicon and single sequencing reaction as a next step (Beall et al., 2000).

The third method, multi-locus sequence typing (MLST), is based on sequencing of seven housekeeping loci to detect allelic changes. Differences in allelic profiles in isolates are assigned to known or new sequence types (STs) (Enright et al., 2001). Similarly to emm typing the method requires PCR amplification, purification of the product and sequencing. The method is relatively fast and reliable but in case of GAS, requires 14 sequencing reactions per isolate, which significantly increases the costs of typing. Because of the cost of MLST, alternative approaches to detect allelic changes in genes included in MLST scheme are developed, such as PCR assays with high resolution melting curves named Mini-MLST (Richardson et al., 2010).

In this report, we present a rapid and cost effective method to detect virulence factors encoded by GAS and phages/ICE elements integrated into genome using set of multiplex PCR reactions. Described system allows easy, simultaneous detection of 20 GAS virulence 
Table I

Integration sites of phages and ICE elements in sequenced S. pyogenes genomes (Modified after Beres and Musser, 2007)

\begin{tabular}{|c|c|c|c|c|c|}
\hline $\begin{array}{l}\text { Integra- } \\
\text { tion site }\end{array}$ & Strain & $\begin{array}{l}\text { Exogenous } \\
\text { Element }\end{array}$ & $\begin{array}{l}\text { Virulence } \\
\text { Gene(s) }\end{array}$ & $\begin{array}{l}\text { CDS Start of the } \\
\text { integrated element }\end{array}$ & $\begin{array}{l}\text { CDS Stop of the } \\
\text { integrated element }\end{array}$ \\
\hline $\mathrm{A}$ & MGAS10394 & 10394.1 & $\mathrm{sdn}$ & 0020 & 0068 \\
\hline B & MGAS8232 & 8232.1 & speA1 & 0336 & 0394 \\
\hline $\mathrm{C}$ & SF370 & 370.1 & speC-spd1 & 0655 & 0712 \\
\hline $\mathrm{C}$ & MGAS10270 & 10270.1 & speC-spd1 & 0536 & 0598 \\
\hline $\mathrm{C}$ & MGAS10750 & 10750.1 & speC-spd1 & 0560 & 0622 \\
\hline $\mathrm{C}$ & Manfredo & $\operatorname{man} .4$ & speC-spd1 & 1263 & 1322 \\
\hline $\mathrm{C}$ & MGAS2096 & 2096.1 & speC-spd1 & 0553 & 0602 \\
\hline $\mathrm{C}$ & MGAS9429 & 9429.1 & speC-spd1 & 0532 & 0594 \\
\hline $\mathrm{C}$ & MGAS8232 & 8232.2 & speC-spd1 & 0716 & 0779 \\
\hline $\mathrm{D}$ & MGAS10394 & 10394.2 & speA4 & 0733 & 0741 \\
\hline $\mathrm{E}$ & SF370 & 370.2 & speH-speI & 0937 & 1008 \\
\hline $\mathrm{E}$ & MGAS10270 & 10270.2 & spd3 & 0796 & 0853 \\
\hline $\mathrm{E}$ & MGAS10750 & 10750.2 & spd3 & 0831 & 0889 \\
\hline $\mathrm{E}$ & Manfredo & $\operatorname{man} .3$ & speH-speI & 1021 & 1070 \\
\hline $\mathrm{E}$ & MGAS9429 & 9429.2 & speH-speI & 0795 & 0851 \\
\hline $\mathrm{F}$ & MGAS315 & 315.1 & none & 0681 & 0736 \\
\hline $\mathrm{F}$ & SSI & SPsP5 & none & 0877 & 0937 \\
\hline $\mathrm{G}$ & SF370 & 370-RD.1 & srtA & 1075 & 1088 \\
\hline G & MGAS5005 & 5005-RD.1 & srtA & 0797 & 0816 \\
\hline G & MGAS10270 & 10270-RD.1 & srtA & 0910 & 0932 \\
\hline G & MGAS10750 & 10750-RD.1 & srtA & 0945 & 0967 \\
\hline G & MGAS2096 & 2096-RD.1 & srtA & 0869 & 0890 \\
\hline G & MGAS9429 & 9429-RD.1 & srtA & 0911 & 0934 \\
\hline $\mathrm{G}$ & MGAS6180 & 6180-RD.0 & srtA & 0771 & 0793 \\
\hline $\mathrm{H}$ & MGAS5005 & 5005.1 & speA2 & 0995 & 1052 \\
\hline $\mathrm{H}$ & MGAS315 & 315.2 & ssa & 0919 & 0978 \\
\hline $\mathrm{H}$ & SSI & SPsP6 & ssa & 1118 & 1172 \\
\hline $\mathrm{H}$ & MGAS10394 & 10394.3 & speK-sla & 0982 & 1026 \\
\hline $\mathrm{H}$ & MGAS8232 & 8232.3 & speL-speM & 1238 & 1309 \\
\hline $\mathrm{H}$ & MGAS6180 & 6180.1 & speC-spd1 & 0967 & 1033 \\
\hline I & MGAS2096 & 2096-RD.2 & tet $(\mathrm{O})$ & 1103 & 1159 \\
\hline $\mathrm{I}$ & MGAS6180 & 6180-RD.1 & none & 1079 & 1089 \\
\hline $\mathrm{J}$ & MGAS10394 & 10394.4 & mef(A), R6 & 1123 & 1173 \\
\hline K & SF370 & 370.3 & $\mathrm{spd} 3$ & 1436 & 1488 \\
\hline K & MGAS5005 & 5005.2 & spd3 & 1168 & 1222 \\
\hline K & MGAS315 & 315.3 & spd4 & 1094 & 1145 \\
\hline $\mathrm{K}$ & SSI & SpsP4 & spd4 & 0717 & 0771 \\
\hline $\mathrm{K}$ & MGAS10750 & 10750.3 & ssa & 1276 & 1328 \\
\hline $\mathrm{K}$ & Manfredo & $\operatorname{man} .2$ & spd4 & 0631 & 0692 \\
\hline K & MGAS10394 & 10394.5 & speC-spd1 & 1194 & 1242 \\
\hline $\mathrm{K}$ & MGAS8232 & 8232.4 & $\operatorname{spd} 3$ & 1444 & 1506 \\
\hline $\mathrm{L}$ & MGAS10270 & 10270.3 & speK-sla & 1297 & 1361 \\
\hline $\mathrm{L}$ & MGAS315 & 315.4 & speK-sla & 1203 & 1266 \\
\hline $\mathrm{L}$ & SSI & SPsP3 & speK-sla & 0597 & 0659 \\
\hline $\mathrm{L}$ & MGAS6180 & 6180.2 & speK-sla & 1220 & 1285 \\
\hline M & MGAS10270 & 10270-RD.2 & $\mathrm{R} 28$ & 1378 & 1411 \\
\hline
\end{tabular}


Table I continued

\begin{tabular}{|c|c|c|c|c|c|}
\hline $\begin{array}{l}\text { Integra- } \\
\text { tion site }\end{array}$ & Strain & $\begin{array}{l}\text { Exogenous } \\
\text { Element }\end{array}$ & $\begin{array}{l}\text { Virulence } \\
\text { Gene(s) }\end{array}$ & $\begin{array}{l}\text { CDS Start of the } \\
\text { integrated element }\end{array}$ & $\begin{array}{l}\text { CDS Stop of the } \\
\text { integrated element }\end{array}$ \\
\hline $\mathrm{M}$ & MGAS6180 & 6180-RD.2 & $\mathrm{R} 28$ & 1302 & 1337 \\
\hline $\mathrm{N}$ & MGAS315 & 315.5 & speA3 & 1300 & 1354 \\
\hline $\mathrm{N}$ & SSI & SPsP2 & speA3 & 0507 & 0561 \\
\hline $\mathrm{N}$ & Manfredo & $\operatorname{man} .1$ & spd3 & 0471 & 0535 \\
\hline $\mathrm{N}$ & MGAS10394 & 10394.6 & sda & 1338 & 1366 \\
\hline $\mathrm{O}$ & MGAS315 & 315.6 & $\operatorname{sdn}$ & 1408 & 1458 \\
\hline $\mathrm{O}$ & SSI-1 & SPsP1 & $\operatorname{sdn}$ & 0408 & 0456 \\
\hline $\mathrm{P}$ & MGAS5005 & 5005.3 & sda & 1414 & 1467 \\
\hline $\mathrm{P}$ & MGAS2096 & 2096.2 & sda & 1440 & 1492 \\
\hline $\mathrm{P}$ & MGAS9429 & 9429.3 & sda & 1415 & 1468 \\
\hline $\mathrm{P}$ & MGAS8232 & 8232.5 & sda & 1745 & 1808 \\
\hline $\mathrm{R}$ & MGAS10394 & 10394.7 & $\operatorname{spd} 3$ & 1540 & 1562 \\
\hline$S$ & MGAS10750 & 10750-RD.2 & $\operatorname{erm}(\mathrm{A})$ & 1679 & 1719 \\
\hline $\mathrm{T}$ & SF370 & 370.4 & none & 2122 & 2147 \\
\hline $\mathrm{T}$ & MGAS10270 & 10270.4 & none & 1874 & 1896 \\
\hline $\mathrm{T}$ & MGAS10750 & 10750.4 & none & 1897 & 1921 \\
\hline $\mathrm{T}$ & Manfredo & $\operatorname{man} .5$ & none & 1764 & 1779 \\
\hline $\mathrm{T}$ & MGAS10394 & 10394.8 & none & 1804 & 1824 \\
\hline $\mathrm{T}$ & MGAS6180 & 6180.3 & none & 1789 & 1813 \\
\hline $\mathrm{U}$ & MGAS10270 & 10270.5 & none & 1917 & 1951 \\
\hline $\mathrm{U}$ & MGAS6180 & 6180.4 & none & 1840 & 1864 \\
\hline
\end{tabular}

factors (VF) and screening of 21 phage and ICE integration sites. The described PCR based method combined with emm typing can be effectively used to differentiate between GAS strains.

\section{Experimental}

\section{Materials and Methods}

Bacterial isolates. Over 650 highly diverse GAS isolates analyzed in the study were sent to KORLD (National Reference Center for Antibiotic Resistance) and KOROUN (National Reference Center for Infections of Central Nervous System) as a part of routine reference activity and as a part of BiNet network for monitoring invasive infections (http://www.koroun. edu.pl/binet_info01.php). Bacterial strains were sent from over 60 laboratories located in multiple geographical areas of Poland and were isolated from various forms of the GAS diseases (throat, skin and invasive infections). Emm types of the strains were determined as routine part of diagnostic work according to (Beall et al., 1996) and CDC's recommendations. In addition, we used highly clonal population of strains which PFGE patterns, emm and ST types were previously determined (Szczypa et al., 2004).
PFGE. PFGE analysis was performed according to modified method by Stanley and co-workers (Stanley et al., 1995). Briefly, agarose plugs containing bacteria were incubated for $4 \mathrm{~h}$ at $37^{\circ} \mathrm{C}$ in lysis buffer with lysozyme $(100 \mu \mathrm{g} / \mathrm{ml}$, Sigma $)$ and mutanolysin $(40 \mu \mathrm{g} / \mathrm{ml}$, Sigma), followed by overnight treatment with proteinase $\mathrm{K}(1 \mathrm{mg} / \mathrm{ml})$. DNA embedded in plugs was digested with SmaI (Fermentas) for $4 \mathrm{~h}$, and separated at $14^{\circ} \mathrm{C}$ for $22 \mathrm{~h}$ in CHEF-DR III system (Bio-Rad) in $0.5 \mathrm{x}$ TBE buffer, with $6 \mathrm{~V} / \mathrm{cm}$, initial pulse $1 \mathrm{~s}$., final pulse $30 \mathrm{~s}$.

Isolation of chromosomal DNA. Chromosomal DNA was isolated from cells grown overnight on Columbia agar plates supplemented with 5\% sheep blood (BioRad, BioMerieux) using the Genomic Mini AX BACTERIA kit (A\&A Biotechnology) or the Genomic Mini kit (A\&A Biotechnology) according to the manufacturer's protocol, with additional initial cell wall digestion with lysozyme $(1 \mathrm{mg} / \mathrm{ml}$, Sigma) and mutanolysin $\left(500 \mathrm{U} / \mathrm{ml}\right.$, Sigma) for $30 \mathrm{~min}$ at $37^{\circ} \mathrm{C}$, in the presence of RNAse. Chromosomal DNA used as a template for PCR reactions was diluted 10-fold.

Primer design and specificity tests. Primer pairs were designed using the modified Primer3 software, available as the Primer-BLAST tool at NCBI (http:// www.ncbi.nlm.nih.gov/tools/primer-blast/). Primers were designed to conserved regions of detected genes (in case of virulence factors) or conserved regions 
Table II

Primers used in this study

\begin{tabular}{|c|c|c|c|c|}
\hline Name & Sequence & $\begin{array}{l}\text { Size } \\
\text { (bp) }\end{array}$ & $\begin{array}{c}\text { Position in reference } \\
\text { sequence }\end{array}$ & Ref. sequence \\
\hline \multicolumn{5}{|c|}{ Toxins MIX I } \\
\hline SpeL F & CCTGAGCCGTGAAATTCCCA & \multirow{2}{*}{657} & $1041734-1041753$ & \multirow{2}{*}{ NC_003485 } \\
\hline SpeL R & ACACCAGAATTGTCGTTTGGT & & $1042370-1042390$ & \\
\hline SpeK F & CCTTGTGTGTGTATCGCTTGC & \multirow{2}{*}{568} & $39278-39298$ & \multirow{2}{*}{ NC_004587 } \\
\hline SpeK R & TTGCTGTCCCCCATCAAACT & & $39825-39844$ & \\
\hline SpeM F & ATCGCTCATCAAACTTTTCCT & \multirow{2}{*}{496} & $1042875-1042895$ & \multirow{2}{*}{ NC_003485 } \\
\hline SpeM R & CCTTGTGTGTGTATCGCTTGC & & $1043350-1043370$ & \\
\hline SpeC F & GCCAATTTCGATTCTGCCGC & \multirow{2}{*}{405} & 617333-617352 & \multirow{2}{*}{ NC_003485 } \\
\hline SpeC R & TGCAGGGTAAATTTTTCAACGACA & & $617715-617737$ & \\
\hline SmeZ F & TTTCTCGTCCTGTGTTTGGA & \multirow{2}{*}{246} & $1662332-1662351$ & \multirow{2}{*}{ NC_007297 } \\
\hline SmeZ R & TTCCAATCAAATGGGACGGAGAACA & & $1662554-1662578$ & \\
\hline SpeI F & TTCATAGACGGCGTTCAACAA & \multirow{2}{*}{176} & $819507-819527$ & \multirow{2}{*}{ NC_002737 } \\
\hline SpeI R & TGAAATCTAGAGGAGCGGCCA & & $819662-819682$ & \\
\hline \multicolumn{5}{|c|}{ Toxins MIX II } \\
\hline ssa F & AAGAATACTCGTTGTAGCATGTGT & \multirow{2}{*}{678} & $39833-39856$ & \multirow{2}{*}{ NC_004585 } \\
\hline ssa R & AATATTGCTCCAGGTGCGGG & & $40491-40510$ & \\
\hline SpeA F & AGGTAGACTTCAATTTGGCTTGTGT & \multirow{2}{*}{576} & $331570-331594$ & \multirow{2}{*}{ NC_003485 } \\
\hline SpeA R & GGGTGACCCTGTTACTCACGA & & $332125-332145$ & \\
\hline SpeH F & TGAGATATAATTGTCGCTACTCACAT & \multirow{2}{*}{480} & $786364-786389$ & \multirow{2}{*}{ NC_011375 } \\
\hline SpeH R & CCTGAGCGGTTACTTTCGGT & & $786824-786843$ & \\
\hline SpeG F & TGGAAGTCAATTAGCTTATGCAG & \multirow{2}{*}{384} & $183579-183601$ & \multirow{2}{*}{ NC_004070 } \\
\hline SpeG R & GCGAACAACCTCAGAGGGCAAA & & $183942-183962$ & \\
\hline SpeJ F & TCCTTGTACTAGATGAGGTTGCAT & \multirow{2}{*}{286} & $364343-364366$ & \\
\hline SpeJ R & GGTGGGGTTACACCATCAGT & & $364609-364628$ & $10+007230$ \\
\hline & & & & \\
\hline $\operatorname{spd} 3 \mathrm{~F}$ & ATCGTCGTACTTGGCAAGGTT & 784 & $1146098-1146118$ & NC $C_{1}$ \\
\hline $\operatorname{spd} 3 \mathrm{R}$ & GCCGCTTCTTCAAACTCTTCG & 104 & $1146861-1146881$ & $1 \mathrm{Ne}=001291$ \\
\hline $\operatorname{sdc} \mathrm{F}$ & AAGCTTAGAAACTCTCTCGCCA & 600 & $49-70$ & AF410852 \\
\hline sdc $\mathrm{R}$ & AGTTCCAGTAATAGCGTTTTTCCGT & 000 & $624-648$ & 月11 410032 \\
\hline sdaB F & TATAGCGCATGCCGCCTTTT & 440 & 1700383-1700402 & NC 007297 \\
\hline sdaB R & TGATGGCGCAAGCAAGTACC & 440 & $1700803-1700822$ & \\
\hline sdaD F & TTTACGCTGAATCGGGCACT & 295 & $1385864-1385883$ & NC 007297 \\
\hline sdaD R & GGCTCTGGTTTGCTTTCCCA & & $1386139-1386158$ & 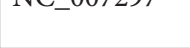 \\
\hline & Prote: & & & \\
\hline speB_F & AGACGGAAGAAGCCGTCAGA & 952 & $1698752-1698771$ & NC 002737 \\
\hline speB_R & TCAAAGCAGGTGCACGAAGC & & $1699684-1699703$ & \\
\hline spyCEP F & GATCCGGCCCATCAAAGCAT & 786 & $344582-344601$ & AF00409? \\
\hline spyCEP R & AGCTGCCACTGATGTTGGTG & & $345348-345367$ & \\
\hline scpA F & GCTCGGTTACCTCACTTGTCC & 622 & $1669854-1669874$ & NC 009332 \\
\hline scpA R & CAATAGCAGCAAACAAGTCACC & & $1670453-1670477$ & \\
\hline Mac F & TCTTGCCCTGTTGAAAGTGT & 389 & $681947-681966$ & NC 011375 \\
\hline Mac R & CGAGGTGGTATTTTTGACGCC & (3) & $682315-682335$ & נN \\
\hline $\operatorname{sic} \mathrm{F}$ & TTACGTTGCTGATGGTGTATATGGT & 150 & $1682672-1682696$ & NC 002737 \\
\hline sic $\mathrm{R}$ & TTTGATAGAGGGTTTTCAGCTGGC & 100 & $1682798-1682821$ & 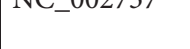 \\
\hline & & & & \\
\hline phageA_F & AGCTTCGTCAGTTCATTGATGAGT & 343 & $34380-34403$ & NC 007297 \\
\hline phageA_R & GGAGTTAATCTTTGTCTGATCACCGT & & $34723-34698$ & \\
\hline
\end{tabular}


Table II continued

\begin{tabular}{|c|c|c|c|c|}
\hline Name & Sequence & $\begin{array}{l}\text { Size } \\
\text { (bp) }\end{array}$ & $\begin{array}{c}\text { Position in reference } \\
\text { sequence }\end{array}$ & Ref. sequence \\
\hline phageG_F & ACTTGAAGAAGCTGGAGCAACA & \multirow{2}{*}{477} & $809606-809627$ & \multirow{2}{*}{ NC_004070 } \\
\hline phageG_R & AGGCAATAGCATCTGGCGTC & & $810052-810033$ & \\
\hline phageB_F & ATCAGTCGCGCCTACCGTAT & \multirow{2}{*}{636} & $301872-301891$ & \multirow{2}{*}{ NC_007297 } \\
\hline phageB_R & TTACTAGAAGGGGCCTGCCG & & $302508-302489$ & \\
\hline phageE_F & TGAGACATGGTGGAAAGCAGA & \multirow{2}{*}{1022} & 739495-739515 & \multirow{2}{*}{ NC_007297 } \\
\hline phageE_R & TGGTCGAAATAACCAAGGGCA & & 740517-740497 & \\
\hline phageD_F & ACGCTTGACTGACTTCGGTG & \multirow{2}{*}{1168} & 720561-720580 & \multirow{2}{*}{ NC_007297 } \\
\hline phageD_R & TGGGACTTATCCGTTGTCACG & & $721729-721709$ & \\
\hline \multicolumn{5}{|c|}{ Phages MIX2 } \\
\hline phageK_F & TGGTCTGCCATCCATTGTCT & \multirow{2}{*}{425} & $1195963-1195982$ & \multirow{2}{*}{ NC_008022 } \\
\hline phageK_R & AGCCTTCAAAGCTGGTAAAGCT & & $1196377-1196356$ & \\
\hline phageJ_F & TGATCCATGGTGACCTGCTT & \multirow[t]{2}{*}{563} & $1123319-1123338$ & \multirow[t]{2}{*}{ NC_007297 } \\
\hline phageJ_R & TCGACATTGGCCAGGGAGAT & & $1123881-1123862$ & \\
\hline phageC_F & ATTGCAACAGGTAGCCCAGC & \multirow{2}{*}{670} & $531240-531259$ & \multirow{2}{*}{ NC_007297 } \\
\hline phageC_R & CTTCACGCGCAGAACGGATA & & $531910-531891$ & \\
\hline phageH_F & AGGCTTTTGAATTACGTTTTGTC & \multirow{2}{*}{870} & $1058226-1058248$ & \multirow{2}{*}{ NC_008022 } \\
\hline phageH_R & TGAATCAGACGGTTGAGGCT & & $1059095-1059076$ & \\
\hline phageM_F & CCACAGCTGTTTCAACACTTTCA & \multirow{2}{*}{1143} & $1252437-1252459$ & \multirow{2}{*}{ NC_007297 } \\
\hline phageM_R & AATTGGCGCTCGGACATGAT & & $1253579-1253560$ & \\
\hline \multicolumn{5}{|c|}{ Phages MIX3 } \\
\hline phageN_F & TCACCGTTAATTCCCATTCGCT & \multirow{2}{*}{349} & $1282773-1282794$ & \multirow{2}{*}{ NC_007297 } \\
\hline phageN_R & CCGTAGGACAGTTGGGCAAA & & $1283121-1283102$ & \\
\hline phageO_F & TCACAAAAGCCAGTTGGTCGAT & \multirow{2}{*}{452} & $1341889-1341910$ & \\
\hline phageO_R & TATCGTCGTGACTACCGGCT & & $1342340-1342321$ & 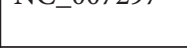 \\
\hline phageP_F & CTAAGGATGTAGTCACTACCCATTTTGTC & 544 & $1493473-1493501$ & \\
\hline phageP_R & TCTGGCTTGACTTACACGCT & 传 & 1494016-1493997 & 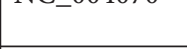 \\
\hline phageI_F & GGTGCCACGTAATGATAACTTGTTC & 666 & $1069901-1069925$ & NC 007297 \\
\hline phageI_R & GTAGACCCGCCACGAAAAGG & & $1070566-1070547$ & \\
\hline phageL_F & GCCAACTGGCCATTTTCTGC & 899 & $1236869-1236888$ & NC 007297 \\
\hline phageL_R & AAGCAAGGAAATGATCGCGG & & $1237767-1237748$ & \\
\hline & Phages MIX4 & & & \\
\hline phageQ_F & CCAGCCATAATCTCAGTTGAGACAGTTG & 364 & $1434160-1434187$ & NC 007297 \\
\hline phageQ_R & GGTTCCATCCAAATCAATGGCAATC & & $1434523-1434499$ & \\
\hline phageR_F & AACGACGTTGCCCTTCCGCA & 432 & $1512239-1512258$ & NC 007297 \\
\hline phageR_R & TCCAAGCTCCTGGCTCGAATGT & 102 & $1512670-1512649$ & 18 \\
\hline phageT_F & CGCTGGCCTTTCTACAACTTCACCA & 555 & $1772901-1772925$ & NC 007297 \\
\hline phageT_R & AGCAACGCTTGAAAAAGATGGCGAT & & $1773455-1773431$ & \\
\hline phageU_F & СTCTTCCCTTTTGTCTGCTAACGGT & 671 & $1796895-1796919$ & NC 007297 \\
\hline phageU_R & CCACGGTCACATCCTTGTTGACGG & 列 & $1797565-1797542$ & 120 \\
\hline phageS_F & ACACTGACCTTTGAAAAACTCATCCA & 917 & $1586507-1586532$ & NC 007297 \\
\hline phageS_R & ATGATAATAGTCGTAGGGATGCTTGTATTATAAAA & & $1587423-1587389$ & \\
\hline & Primers to detect integratio & nto F site & & \\
\hline PhageF F & CCCGAAGTGAAATCGATGATTGACA & $\sim 1000-$ & 778913-778937 & NC_007297 \\
\hline 1 10 & TCCCACGCTCACGCTCCAAA & $\sim 3000$ & $780465-780446$ & NC_007297 \\
\hline & Control primers for phag & tection & & \\
\hline DnaA_F & TGCCGAAGCTATTCGCGCCA & 240 & $1227-1246$ & NC 007297 \\
\hline DnaA_R & ACTGTTGAATGGTCTCTGCCACCA & & $1466-1443$ & \\
\hline
\end{tabular}


Table III

PCR composition of the multiplex reactions

\begin{tabular}{|l|c|c|c|c|c|c|}
\hline \multicolumn{1}{|c|}{ Reagent } & Toxins MIX I & $\begin{array}{c}\text { Toxins MIX II } \\
\text { and proteases }\end{array}$ & DNAses & $\begin{array}{c}\text { Phages MIX 1,2 } \\
\text { and 4 }\end{array}$ & $\begin{array}{c}\text { Phages } \\
\text { MIX 3 }\end{array}$ & Phage F \\
\hline $100 \mu \mathrm{M}$ or $10 \mu \mathrm{M}$ primers mix & $0.6 \mu \mathrm{l}$ & $0.5 \mu \mathrm{l}$ & $0.4 \mu \mathrm{l}$ & $0.6 \mu \mathrm{l}$ & $0.7 \mu \mathrm{l}$ & $0.2 \mu \mathrm{l}$ \\
\hline $\begin{array}{l}10 \mathrm{x} \text { Taq polymerase buffer } \\
\text { with }\left(\mathrm{NH}_{4}\right)_{2} \mathrm{SO}_{4} \text { (Fermentas) }\end{array}$ & $0.5 \mu \mathrm{l}$ & $0.5 \mu \mathrm{l}$ & $0.5 \mu \mathrm{l}$ & $0.5 \mu \mathrm{l}$ & $0.5 \mu \mathrm{l}$ & $0.5 \mu \mathrm{l}$ \\
\hline $25 \mathrm{mM} \mathrm{MgCl}_{2}$ & $0.5 \mu \mathrm{l}$ & $0.5 \mu \mathrm{l}$ & $0.5 \mu \mathrm{l}$ & $0.5 \mu \mathrm{l}$ & $0.5 \mu \mathrm{l}$ & $0.5 \mu \mathrm{l}$ \\
\hline $1 \mathrm{mM} \mathrm{dNTP}$ & $0.5 \mu \mathrm{l}$ & $0.5 \mu \mathrm{l}$ & $0.5 \mu \mathrm{l}$ & $0.5 \mu \mathrm{l}$ & $0.5 \mu \mathrm{l}$ & $0.5 \mu \mathrm{l}$ \\
\hline water & --- & --- & --- & --- & --- & $2.15 \mu \mathrm{l}$ \\
\hline $\begin{array}{l}10 \mathrm{x} \text { diluted chromosomal } \\
\text { DNA template }\end{array}$ & $2.8 \mu \mathrm{l}$ & $2.9 \mu \mathrm{l}$ & $3.0 \mu \mathrm{l}$ & $2.8 \mu \mathrm{l}$ & $2.7 \mu \mathrm{l}$ & $1 \mu \mathrm{l}$ \\
\hline Taq polymerase (Fermentas) & $0.1 \mu \mathrm{l}(0.5 \mathrm{U})$ & $0.1 \mu \mathrm{l}(0.5 \mathrm{U})$ & $0.1 \mu \mathrm{l}(0.5 \mathrm{U})$ & $0.1 \mu \mathrm{l}(0.5 \mathrm{U})$ & $0.1 \mu \mathrm{l}(0.5 \mathrm{U})$ & $0.15 \mu \mathrm{l}(0.75 \mathrm{U})$ \\
\hline
\end{tabular}

of genes surrounding phage integration site. Primer sequences, their chromosomal location and accession number of reference sequence are listed in Table II.

Composition of the reaction and PCR conditions. To detect 20 virulence factors, four multiplex reactions were designed. To detect 21 mobile genetic element integration sites, four multiplex and one singleplex reaction were designed. Composition of all primer mixes and size of the amplicons generated in multiplex reactions are listed in Table II. For the ease of use, equal volumes of $100 \mu \mathrm{M}$ primer stocks were mixed into appropriate mixes, namely: "Toxins MIX I", “Toxins MIX II", "proteases", "DNAses". For the case of use, equal volumes of $10 \mu \mathrm{M}$ stocks were mixed into: "Phages MIX 1", "Phages MIX 2", "Phages MIX 3" and "Phages MIX 4". To avoid degradation, primers premixes were aliquoted, so the single portion was sufficient to run the whole 96 well PCR plate without multiple freezing-thawing cycles. Final composition of each PCR reaction is presented in Table III. All PCR reactions were carried out in a total volume of $5 \mu \mathrm{l}$ in a Veriti thermocycler (Applied Biosystems); conditions of PCR reaction are presented in Table IV.

Statistical Analysis. Simpson's Index of Diversity, and the Wallace Coefficient were calculated using online tool http://darwin.phyloviz.net/ (Carrico et al., 2006; Pinto et al., 2008). Analysis of strains was performed with Bionumerics package (Applied Maths).

Table IV

PCR reaction conditions used to amplify products in multiplex reactions

\begin{tabular}{|l|c|c|c|c|c|c|}
\hline \multirow{2}{*}{} & \multicolumn{2}{|c|}{$\begin{array}{l}\text { Toxins MIX I } \\
\text { and II Dnases }\end{array}$} & \multicolumn{2}{c|}{ Proteases mix } & \multicolumn{2}{c|}{$\begin{array}{c}\text { Phages 1-4 } \\
\text { and phage F }\end{array}$} \\
\cline { 2 - 7 } & $\mathrm{T}$ & $\mathrm{t}$ & $\mathrm{T}$ & $\mathrm{t}$ & $\mathrm{T}$ & $\mathrm{t}$ \\
\hline Denaturation & $95^{\circ} \mathrm{C}$ & $0: 15$ & $95^{\circ} \mathrm{C}$ & $0: 15$ & $95^{\circ} \mathrm{C}$ & $0: 15$ \\
\hline Annealing & $60^{\circ} \mathrm{C}$ & $0: 20$ & $52.5^{\circ} \mathrm{C}$ & $0: 45$ & $64^{\circ} \mathrm{C}$ & $0: 30$ \\
\hline Elongation & $72^{\circ} \mathrm{C}$ & $2: 00$ & $72^{\circ} \mathrm{C}$ & $3: 00$ & $72^{\circ} \mathrm{C}$ & $3: 30$ \\
\hline
\end{tabular}

All reactions were amplified for 40 cycles with initial denaturation was carried out for $3 \mathrm{~min}$ at $95^{\circ} \mathrm{C}$, and final elongation for $7 \mathrm{~min}$ at $72^{\circ} \mathrm{C}$ $\mathrm{T}$ - temperature; $\mathrm{t}$ - time

\section{Results and Discussion}

Detection of phages. Phages/ICEs of group A Streptococcus are major sources of genetic diversity in this group of organisms, carriers of antibiotic resistance genes and multiple proven and putative virulence factors (Beres and Musser, 2007). Detection of integrated mobile elements can distinguish between GAS strains with closely related genetic backgrounds and with addition of other typing methods can be used in detailed epidemiological investigations (Beres et al., 2010; Beres et al., 2004).

Comparison of multiple GAS genomic sequences revealed 21 potential integration sites for phages and ICE elements (Beres and Musser, 2007) and Table I. To screen all 21 integration sites (named from A through $\mathrm{U}$ as in (Beres and Musser, 2007)), we designed set of four multiplex PCR reactions that are able to amplify products only when no element is integrated between open reading frames flanking integration site. Detection of the integrated phages and ICE elements is based on the assumption that in the standard PCR reaction, large (above $10 \mathrm{~kb}$ ) element integrated into the chromosome cannot be efficiently amplified and furthermore detected. The designed primer pairs within single multiplex reaction had equal annealing temperatures and $100 \mathrm{bp}$ or more size difference between products for easy product tracing. Primers were tested individually using an annealing gradient of temperatures from 55 to $72^{\circ} \mathrm{C}$ to select the optimal annealing temperature for multiplex PCR. Only primers that generated single amplicons were selected for composition of multiplex reactions (data not shown).

Because the lack of the PCR product denotes positive detection of large integrated element into particular integration site, to avoid PCR errors resulting from negative PCR amplification, in all multiplex reactions positive control of amplification (240 bp fragment of $d n a A$ gene) is included. Examples of phage profile (PP) typing of randomly chosen strains from our GAS collection are 
Table V

Simpson's Index of Diversity (SDI) and Wallace's coefficient (WC), calculated for strains analyzed by phage profiling (PP) and virulence factor profiling (VF)

A.

\begin{tabular}{|l|c|c|c|}
\hline Typing Method & \# partitions & Simpson’s ID & C.I. (95\%) \\
\hline Phage profile (PP) & 185 & 0.965 & $(0.960-0.971)$ \\
\hline Virulence factor profile (VF) & 95 & 0.943 & $(0.936-0.951)$ \\
\hline Virulence factor profile (VF) without "proteases mix" & 94 & 0.944 & $(0.936-0.952)$ \\
\hline emm type & 40 & 0.908 & $(0.899-0.917)$ \\
\hline
\end{tabular}

B.

\begin{tabular}{|l|c|c|c|c|c|}
\hline & PFGE & ST & emm & VF & PP \\
\hline PFGE & & 1.000 & 1.000 & 0.990 & 0.986 \\
& & $(1.000-1.000)$ & $(1.000-1.000)$ & $(0.979-1.000)$ & $(0.974-0.997)$ \\
\hline ST & 0.564 & & 1.000 & 0.899 & 0.648 \\
& $(0.379-0.749)$ & & $(1.000-1.000)$ & $(0.768-1.000)$ & $(0.472-0.824)$ \\
\hline emm & 0.564 & 1.000 & & 0.899 & 0.648 \\
& $(0.379-0.749)$ & $(1.000-1.000)$ & & $(0.768-1.000)$ & $(0.472-0.824)$ \\
\hline VF & 0.622 & 1.000 & 1.000 & & 0.721 \\
& $(0.430-0.813)$ & $(1.000-1.000)$ & $(1.000-1.000)$ & & $(0.556-0.886)$ \\
\hline PP & 0.858 & 1.000 & 1.000 & 1.000 & \\
& $(0.703-1.000)$ & $(1.000-1.000)$ & $(1.000-1.000)$ & $(1.000-1.000)$ & \\
\hline
\end{tabular}

Information about absence (0)/presence (1) of particular virulence factor or integrated element was concatenated into binary sequence of 20 or 21 digits and used for calculations with http://darwin.phyloviz.net/ComparingPartitions/. A SDI calculated for group of 656 divergent strains; B. WC calculated for group of highly clonal strains (PFGE pattern A from Szczypa, et al., 2004)

presented in Figure 1. To test primer specificity, we preformed PP typing using two reference strains of known genomic sequence: MGAS6180 (NC_007296.1 (Green et al., 2005)) and MGAS10270 (NC_008022 (Beres and Musser, 2007)). Based on the genomic sequence, strain MGAS6180 carries 7 elements integrated into sites $G$, H, I, L, M, T, U and MGAS10270 carries 7 elements integrated into sites C, E, G, L, M, T, U (Table I) ( Beres and Musser, 2007). In concordance with the predicted product presence and size, we were able to detect fragments that denote putative integration sites without inserted element, and we were not able to detect products that amplified large integrated element (Fig. 2). In some cases, such as for site G in strain MGAS10270 and site L in strain MGAS6180, very weak bands are observed and are probably a signal derived from a DNA isolated from fraction of GAS cells where the element was excised from the chromosome.

Two mobile elements 315.1 and SPsP5 are integrated into integration site "F" in M3 strain MGAS315 (between ORFs SpyM3_0680 and SpyM3_0737) and SSI-1 (between ORFs SPs0876 and SPs0938), respectively (Beres and Musser, 2007). However, based on the BLAST searches in all sequenced GAS genomes, the region encompassed by ORFs flanking prophage integration site varies in length and gene content in different strains. Therefore, primers detecting integrated element $\mathrm{F}$ amplify fragment of varying size, from about $1 \mathrm{~kb}$ to over $3 \mathrm{~kb}$ in the absence of integrated prophage. We decided to exclude primers detecting integration in the F site from multiplex reaction to increase PCR specificity and efficiency and run the reaction separately (Fig. 1E). With additional optimization of the reaction, primers detecting $\mathrm{F}$ integration site can be included in "phage mix 3", however detected bands are often weaker and gels more difficult for interpretation (Fig. 2).

To determine resolution of the phage profile detection as a typing method, we calculated Simpson's Index of Diversity (SID) based on analysis of highly diverse 656 GAS strains (Table VA). Among $40 \mathrm{emm}$ types, we detected 185 distinct phage profiles with Simpson's Index of Diversity of 0.965 (CI 95\% 0.960-0.971). Phage profile is also good predictor of emm type, with PP $\rightarrow$ emm Wallace's coefficient (WC) equal 0.953 (CI 95\% 0.926-0.980).

Insertion or excision of large DNA fragments such as phages/ICEs from the chromosome usually is reflected in PFGE analysis. To test if the presence of integrated elements correlates with PFGE pattern, we analyzed homogenous population of strains previously described by Szczypa and co-workers (Szczypa et al., 2004). Performed PP analysis showed that detection of elements inserted into putative integration sites correlates with PFGE patterns, emm type and ST (Fig. 3AB). 
A

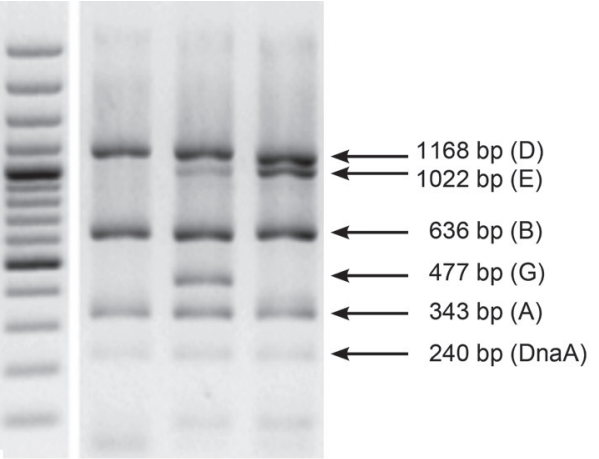

D

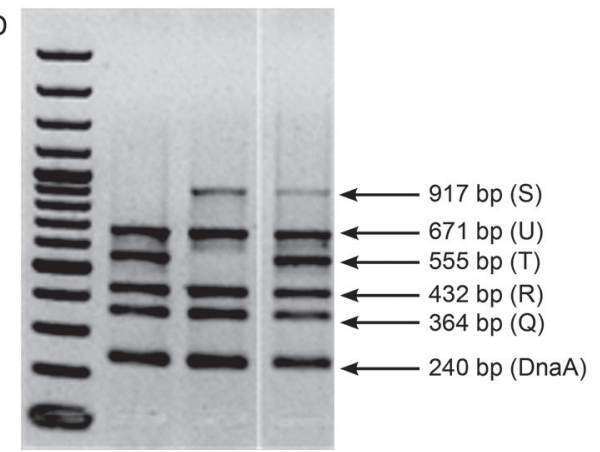

B

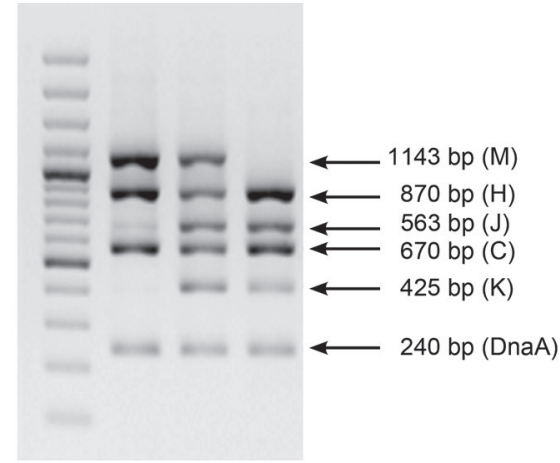

C

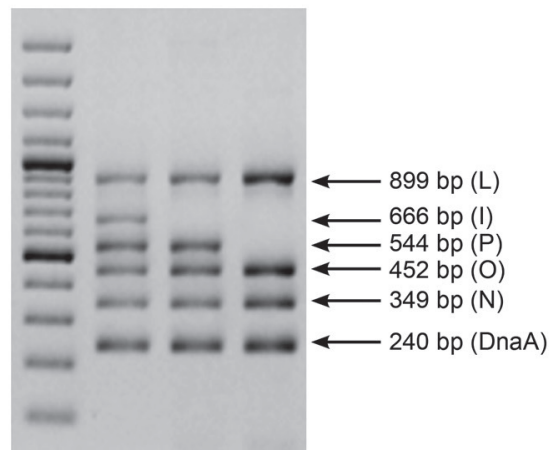

$E$

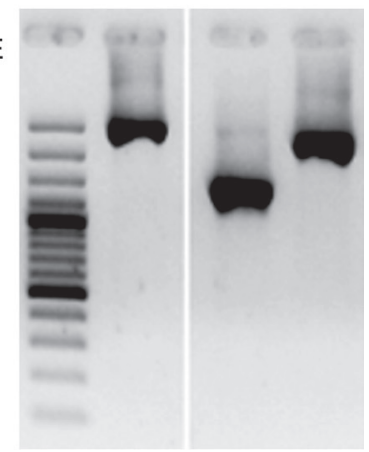

Fig. 1. Detection of twenty one GAS phage and ICE integration sites in randomly chosen GAS strains.

Each panel represents multiplex PCR reaction: A: Phage MIX1, B: Phage MIX2, C: Phage MIX3, D: Phage MIX4, E. Phage F Amplification of a product denotes lack of integrated element at the chromosomal location. Arrows denote expected product size based on the GAS genomic sequences, letters in parentheses denote the mobile element integration sites after (Beres and Musser, 2007) and Table I, 1.5\% agarose/TBE, marker: GeneRuler ${ }^{\text {rm }} 100$ bp Plus DNA Ladder (Fermentas).

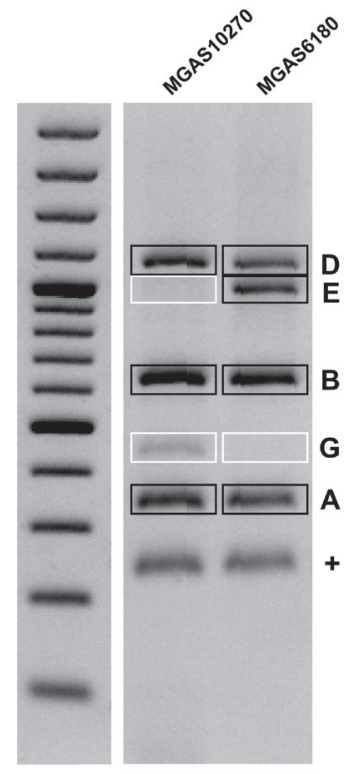

MIXI

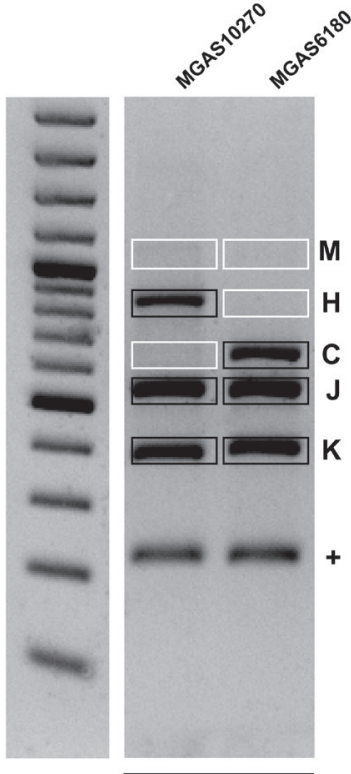

MIX II

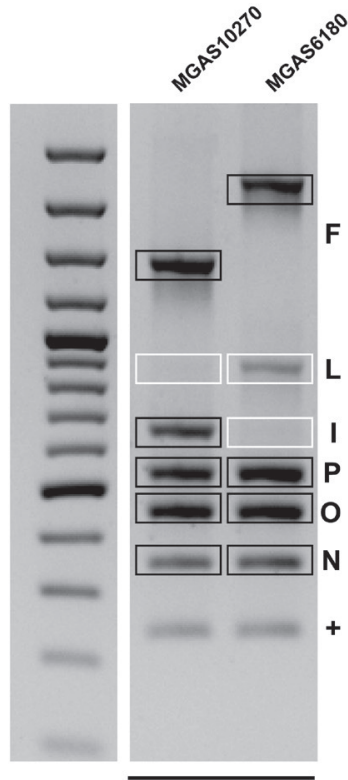

MIX III

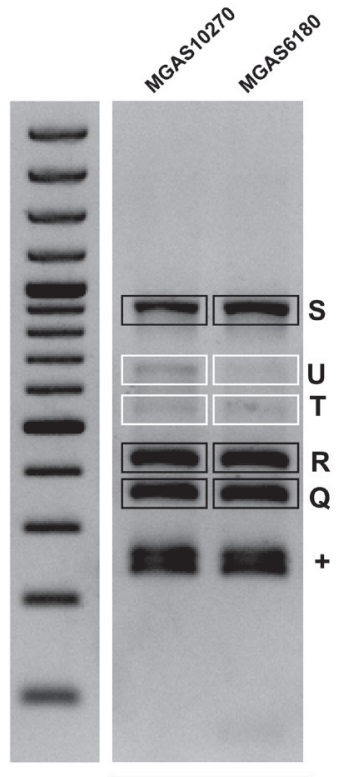

MIX IV

Fig. 2. Detection of integrated mobile genetic elements in reference strains MGAS6180 and MGAS10270.

Capital letters denote the mobile element integration sites (after (Beres and Musser, 2007) and Table I) detected by each multiplex reaction and "+" denotes positive control - amplification of $d n a A$ fragment. Amplification of a product denotes lack of integrated element at the chromosomal location. Black boxes denote locations without integrated element and white boxes denote chromosomal locations with integrated mobile elements as annotated for the genomic sequences of MGAS6180 (sites G, H, I, L, M, T, U) (Green, et al., 2005) and MGAS10270 (sites C, E, G, L, M, T, U). 1.5\% agarose in TBE buffer, marker: GeneRuler ${ }^{\mathrm{mw}} 100 \mathrm{bp}$ Plus DNA Ladder (Fermentas). 


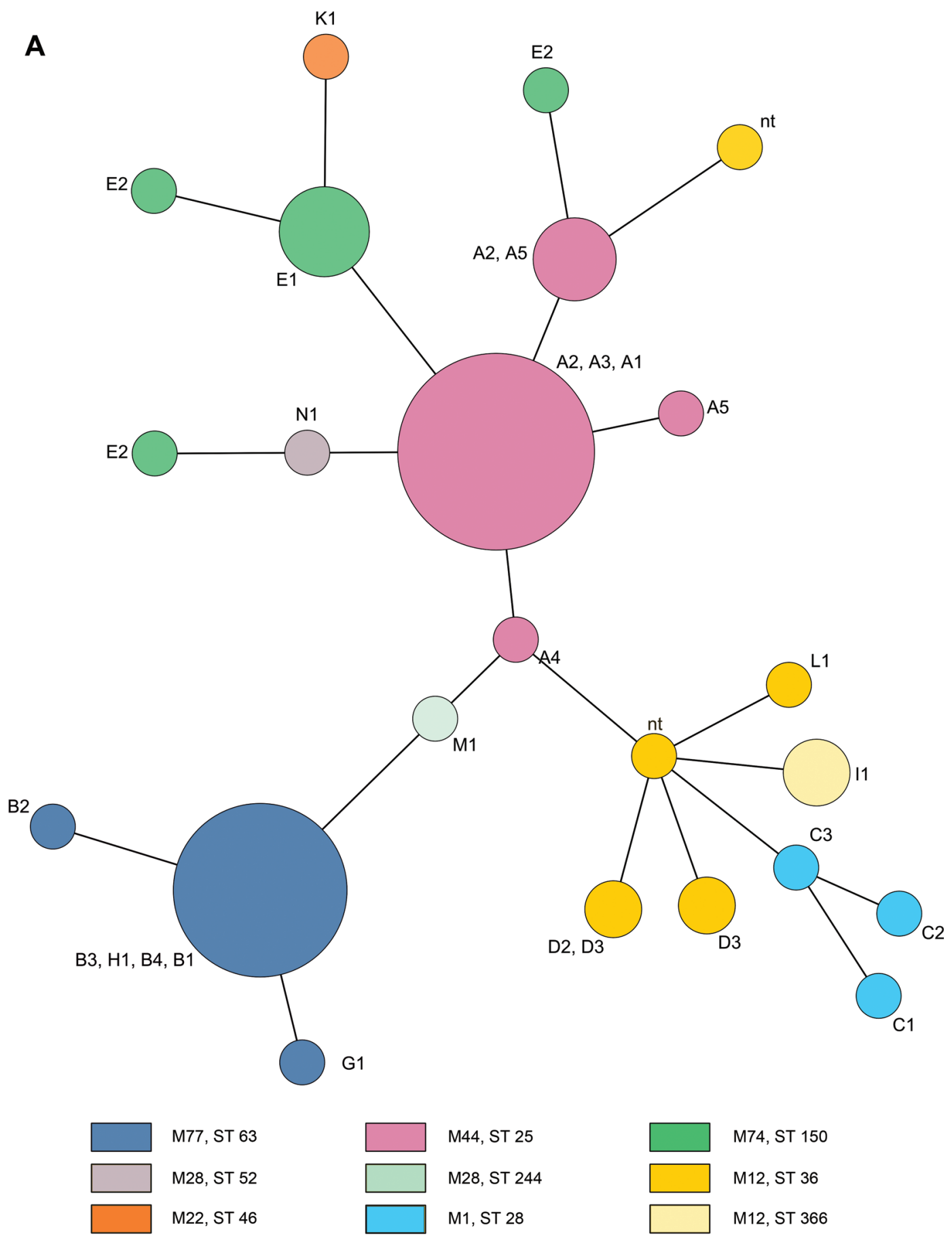

Fig. 3A.

We detected differences in phage content within single $\mathrm{emm} / \mathrm{ST}$ groups that was reflected in described previously subtype of PFGE (Fig. 3). Although PFGE subtyping is the best predictor of phage content $\left(\mathrm{WC}_{\mathrm{PFGE} \rightarrow \mathrm{PP}}=\right.$ 0.986; CI 95\% 0.974-0.997), conversely, PP typing can detect variants that reflect PFGE subtypes with over $85 \%$ probablity $\left(\mathrm{WC}_{\mathrm{PP} \rightarrow \mathrm{PFGE}}=0.858\right.$; CI 95\% $\left.0.703-1.000\right)$ (Table VB).

Detection of virulence factors. Multiple virulence factors produced by GAS such as superantigens, proteases and DNAses are linked to disease severity and clinical manifestations of infection (Bernal et al., 1999;
Fraser et al., 2000; Proft et al., 2000). In particular, presence of speA gene is associated with streptococcal toxic like shock syndrome and scarlet fever (Hauser et al., 1991; Musser et al., 1991; Stevens et al., 1989; Yu and Ferretti, 1989) and smeZ participates in repression of cognate anti-streptococcal responses (Unnikrishnan et al., 2002). Therefore, the detection of virulence factors can be used as a predictor of disease severity and as a diagnostic marker.

We designed set of four, low volume, multiplex reactions that allow simultaneous detection of 20 GAS virulence factors. Two multiplex reactions detect genes 
B

PHAGES/ICES

Virulence Factors

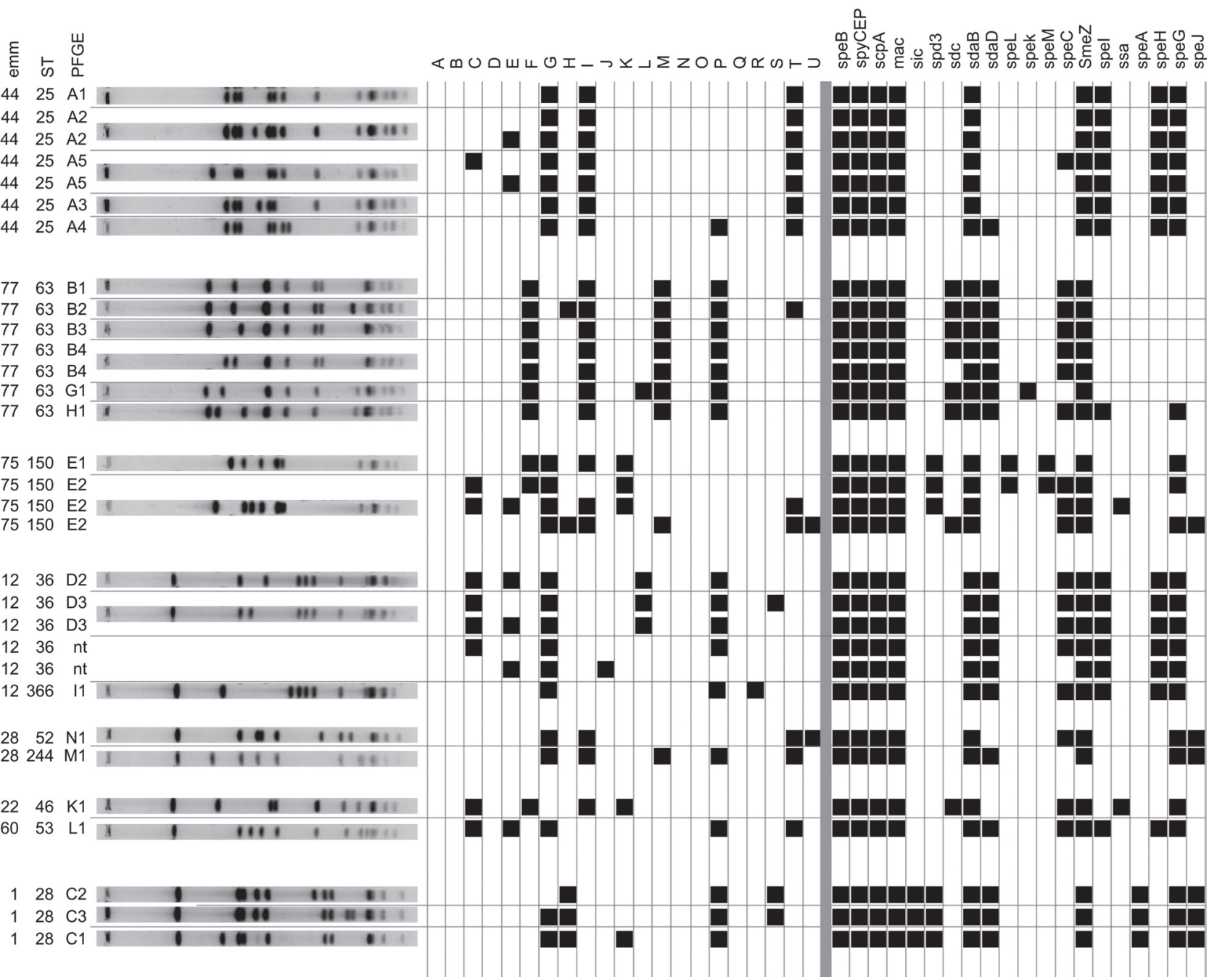

Fig. 3. Correlation between detected phage/ICE integration sites and virulence factors with $\mathrm{M}$ type (emm), sequence type (ST) and PFGE pattern (after (Szczypa et al., 2004)).

A. A through $\mathrm{K}$ designations (with subtypes marked with arabic numerals) denote PFGE patterns detected by (Szczypa et al., 2004). Clusters and relationship between them are based on detected phages and ICE elements and were determined using Minimum Spanning Tree method of BioNumerics package by Applied Maths. Circle size indicates number of isolates in each PFGE group. B. Black rectangles denote phages/ICEs and virulence factors detected in analyzed strains. Strips of PFGE gels represent detected patterns and sub-patterns.

encoding 11 superantigens: speL, speK, speM, speC, smeZ, speI and ssa, speA, speH, speG, speJ; one multiplex PCR detects DNases: chromosomal $s d a B$ (named also streptodornase B, speF, MF, designated M5005_1738 in strain MGAS5005) and phage encoded spd3 (M5005_Spy1169), sdc, (sdalpha, SpyM3_1409), sdaD (M5005_1415); fourth multiplex reaction detects genes encoding proteases $s c p A$, speB, mac, spyCEP and streptococcal inhibitor of complement sic. An example of the PCR products separation after detection of virulence factors in four multiplex reactions is presented in Fig. 4 A-D.

To assure that the possible negative result of amplification of multiplex reactions "Toxins MIX I" and "Toxins MIX II" was not caused by poor quality of DNA, results of the reactions were always cross-checked with the results of other reactions and the detection of chromosomally located genes served as positive control of DNA amplification.

Distribution of phage encoded virulence factors could be in majority of cases attributed to the detected integrated elements known to encode particular virulence factor (Beres and Musser, 2007). Therefore, detection of particular superantigens was routinely compared with detected phage profiles. Example of such comparison can be seen in Fig. 5. Lack of detected products in multiplex reaction "Toxins MIX I" correlates with detection of elements integrated into sites $\mathrm{F}$, $\mathrm{G}$ and $\mathrm{T}$ that do not encode superantigens. In case of the same strain, detection $s p d 3$ gene correlates with the detection of the mobile element integrated into $\mathrm{R}$ site that can carry this type of DNAse. Detection of virulence factors was validated using reference strains of 
A

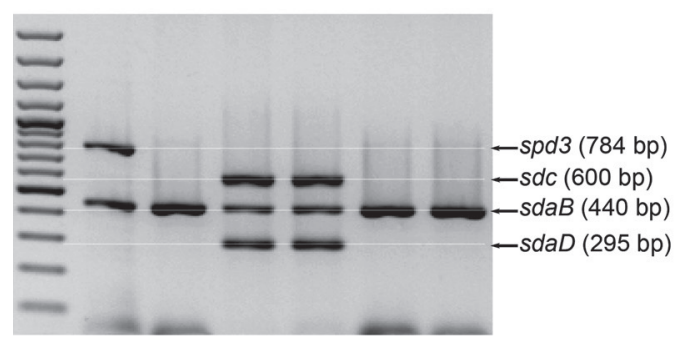

C

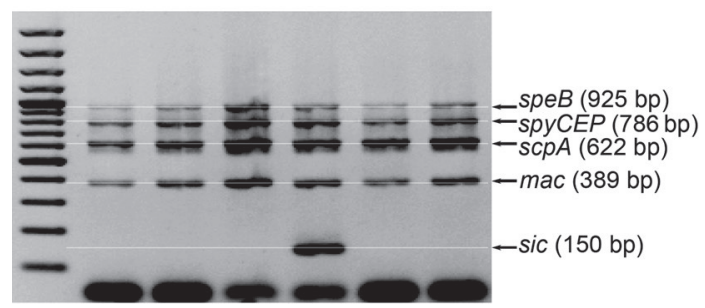

B

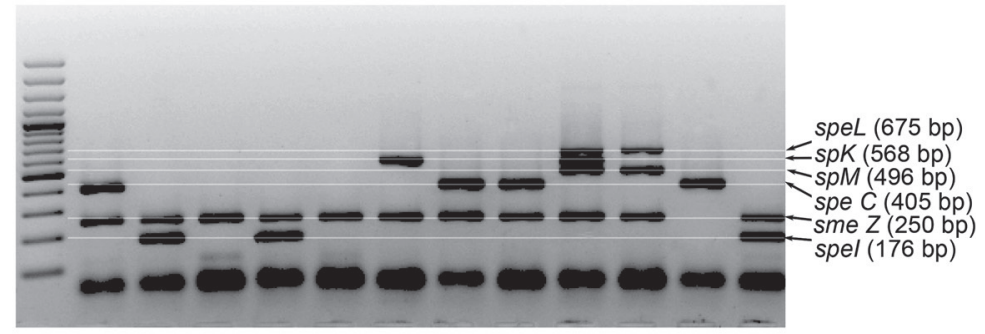

D

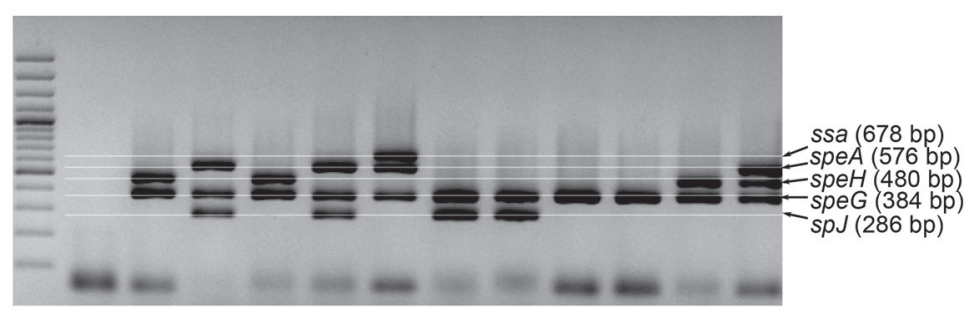

Fig. 4. Detection of twenty GAS virulence factors in randomly chosen strains.

Each panel represents multiplex PCR reactions: A: DNAses, B: toxins I, C: proteases and sic, D: toxins II. 1.5\% agarose/TBE, marker: GeneRuler 100 bp Plus DNA Ladder (Fermentas).
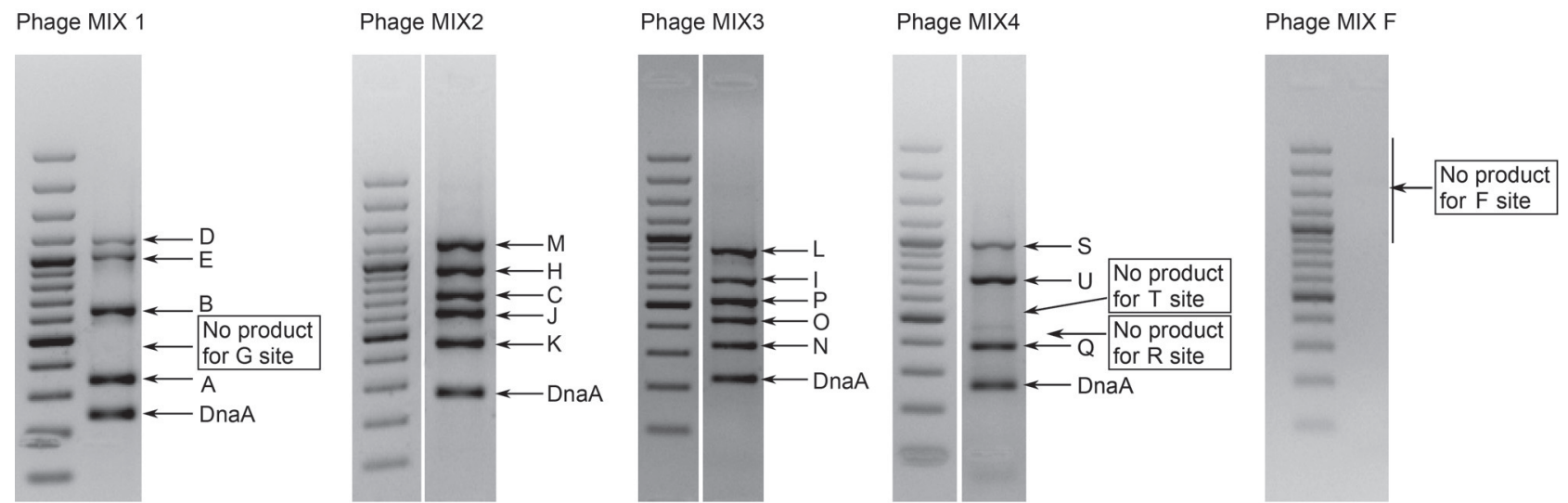

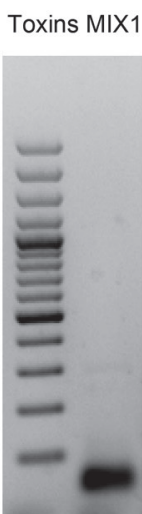

No product detected Lack of tixins: Spel, SpeC SmeZ, SpeK, SpeM, Spel

Detected phages/ICEs integrated into F, G, T, R sites

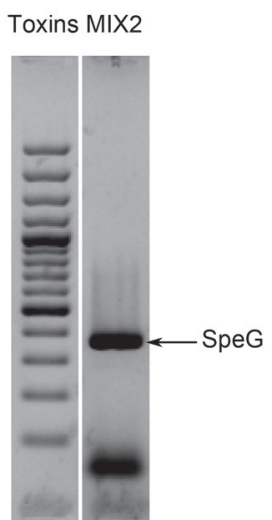

SpeG detected Lack of toxins: SpeJ, SpeH, SpeA, ssa

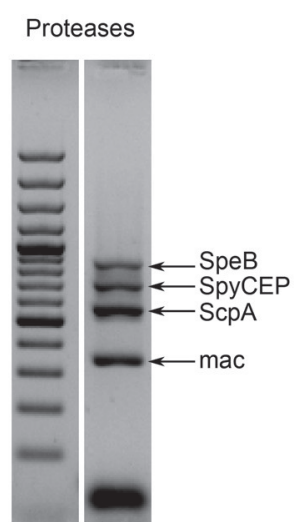

SpeB, SpyCEP, ScpA and Mac detected
DNAses

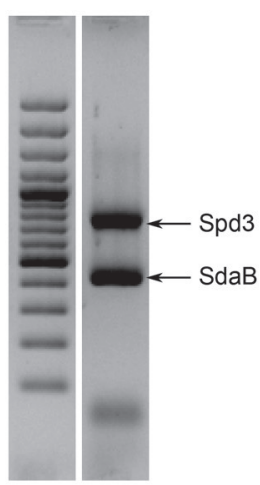

SdaB and Spd3 detected Lack of Sdc and SdaD

Fig. 5. Analysis of phage and virulence factors presence in a single M81 strain.

Analysis of phage integration sites detected elements integrated into F, G, T and R chromosomal locations. Based on the genome sequences, the integration sites correspond with the elements not carrying any virulence factors (sites F, G and T) and encoding Spd3 DNase (site R) (Beres and Musser, 2007). During the analysis of virulence factors, phage encoded spd3 DNAse was detected, as well as chromosomally encoded $s p e G, s p e B, s p y C E P$, scpA, mac and sdaB.Marker: GeneRuler" 100 bp Plus DNA Ladder (Fermentas). 

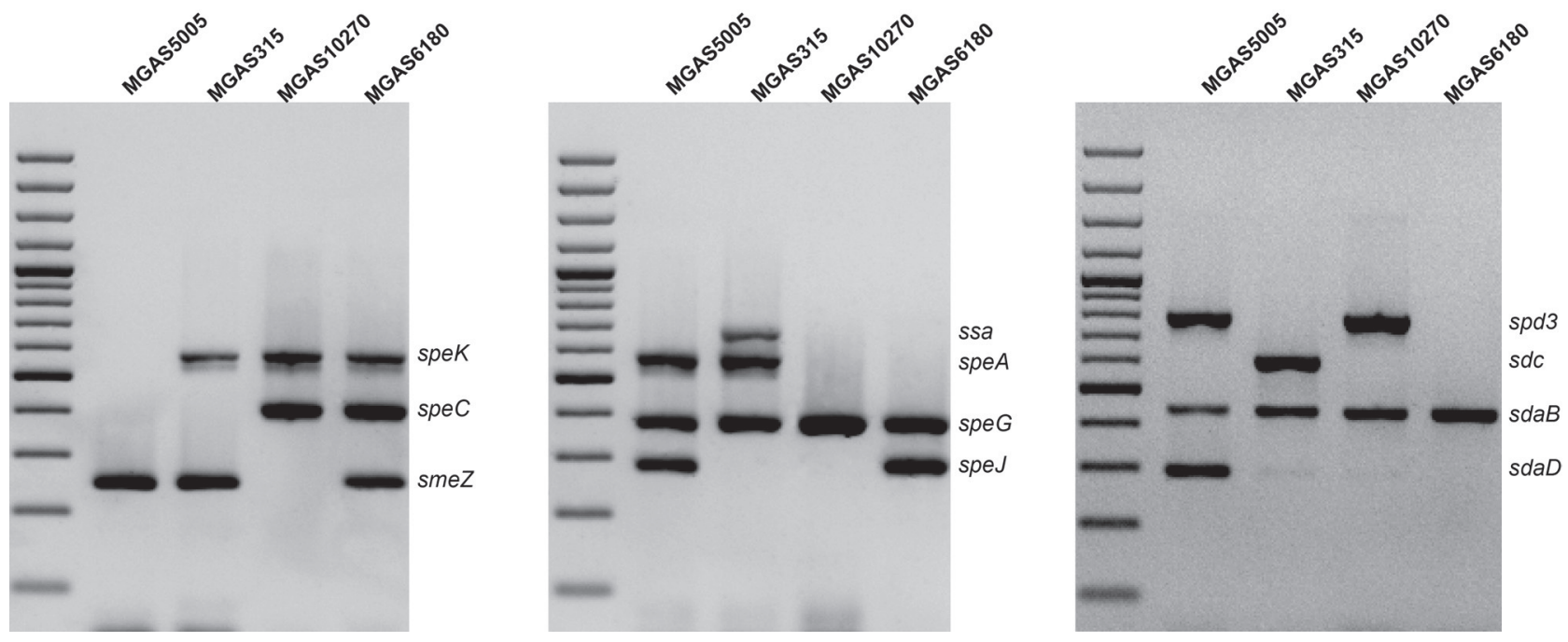

Fig. 6. Detection of toxins and DNAses in sequenced reference GAS strains MGAS5005 (NC_007297.1), MGAS315 (NC_004070.1), MGAS10270 (NC_008022.1) and MGAS6180 (NC_007296.1).

Each panel represents multiplex PCR reaction: A: toxins I , B:, toxins II C: DNAses. Chromosomally located speB, mac, spyCEP were detected in all cases sic gene was detected in MGAS5005 (data not shown). 1.5\% agarose/TBE, marker: GeneRuler ${ }^{\mathrm{mt}} 100$ bp Plus DNA Ladder (Fermentas).

known genomic sequence and virulence factor profiles; the detected profile matched predicted profiles (Fig. 6).

Analysis of 656 diverse GAS strains detected 95 virulence factor profiles among $40 \mathrm{emm}$ types and 185 phage profiles (SID $=0.943$; CI 95\% 0.936-0.951). The number of detected VF profiles is lower than phage profiles because phages encoding certain virulence factors, such as SpeC or SpeK can be carried by phages integrated in various sites (Beres and Musser, 2007), so single virulence factor profiles can mach different phage profiles.

Based on SID calculations (Table V) and the fact that chromosomally encoded proteases SpeB, SpyCEP, ScpA and Mac are detected in virtually all strains, the detection of virulence factors can be simplified. Abbreviated method (without "proteases mix") has identical resolution as not abbreviated method (Table V). The mix, however, can be used for the analysis of emm type 1 strains to detect variants of sic gene. As an alternative approach, primers detecting sic gene can be added to mixes "toxins II" or "DNases".

The group of strains chosen to further test the method of virulence factor detection, was highly clonal based on previous analyses (Szczypa et al., 2004) and this work). Analysis of genes encoding virulence factors shows that these strains have potential to produce almost identical virulence factors within each PFGE group. In addition particular virulence factors within each group seem to be encoded by the same phage and differences in virulence factor profiles are reflected by subgroups of PFGE patterns (Fig. 3B).

In a conclusion, we developed two inexpensive methods that allow easy differentiation between S. pyogenes strains. In addition, detection of superantigens and other virulence factors in clinical strains can provide invaluable information for further epidemiological investigations. Comparing with PFGE and MLST, the method is fast $(2-3 \mathrm{~h}$ of PCR amplification with additional time for electrophoresis) and cost of multiplex PCR reactions is much lower than sequencing. The discriminatory power of the system used as typing method is comparable with PFGE, and it can be used when rapid strain comparison is required.

\section{Acknowledgments}

We are thankful to members of KORLD and KOROUN for strain collection and to Katarzyna Szczypa for critical reading of the manuscript.

The work was supported by: grant N N401 536140 from National Center for Science to W.H.; internal funding (DS.5.82 and DS 5.67) to I.S.; National Program of Antibiotics Protection (NPOA-Moduł 1), unrestricted grant from GlaxoSmithKline Poland and Polish Ministry of Science grant for bacterial collection maintenance - Mikrobank.

\section{Literature}

Beall B., R. Facklam and T. Thompson. 1996. Sequencing emmspecific PCR products for routine and accurate typing of group A streptococci. J. Clin. Microbiol. 34: 953-958.

Beall B., G. Gherardi, M. Lovgren, R.R. Facklam, B.A. Forwick and G.J. Tyrrell. 2000. emm and sof gene sequence variation in relation to serological typing of opacity-factor-positive group A streptococci. Microbiology 146: 1195-1209.

Beres S.B., R.K. Carroll, P.R. Shea, I. Sitkiewicz, J.C. MartinezGutierrez, D.E. Low, A. McGeer, B.M. Willey, K. Green, G.J. Tyrrell, et al. 2010. Molecular complexity of successive bacterial epidemics deconvoluted by comparative pathogenomics. Proc. Natl. Acad. Sci. USA 107: 4371-4376.

Beres S.B. and J.M. Musser. 2007. Contribution of exogenous genetic elements to the group A Streptococcus metagenome. PLoS One. 2: e800. 
Beres S.B., G.L. Sylva, D.E. Sturdevant, C.N. Granville, M. Liu, S.M. Ricklefs, A.R. Whitney, L.D. Parkins, N.P. Hoe, G.J. Adams, et al. 2004. Genome-wide molecular dissection of serotype M3 group A Streptococcus strains causing two epidemics of invasive infections. Proc. Natl. Acad. Sci. USA. 101: 11833-11838.

Bernal A., T. Proft, J.D. Fraser and D.N. Posnett. 1999. Superantigens in human disease. J. Clin. Immunol. 19: 149-157.

Bert F., C. Branger and N. Lambert-Zechovsky. 1997. Pulsed-field gel electrophoresis is more discriminating than multilocus enzyme electrophoresis and random amplified polymorphic DNA analysis for typing pyogenic streptococci. Curr. Microbiol. 34: 226-229.

Carapetis J.R., A.C. Steer, E.K. Mulholland and M. Weber. 2005. The global burden of group A streptococcal diseases. Lancet. Infect. Dis. 5: 685-694.

Carrico J.A., C. Silva-Costa, J. Melo-Cristino, F.R. Pinto, H. de Lencastre, J.S. Almeida and M. Ramirez. 2006. Illustration of a common framework for relating multiple typing methods by application to macrolide-resistant Streptococcus pyogenes. J. Clin. Microbiol. 44: 2524-2532.

Chiang-Ni C. and J.J. Wu. 2008. Effects of streptococcal pyrogenic exotoxin B on pathogenesis of Streptococcus pyogenes. J. Formos. Med. Assoc. 107: 677-685.

Cleary P.P., E.L. Kaplan, C. Livdahl and S. Skjold. 1988. DNA fingerprints of Streptococcus pyogenes are M type specific. J. Infect. Dis. 158: 1317-1323.

Cleary P.P., U. Prahbu, J.B. Dale, D.E. Wexler and J. Handley. 1992. Streptococcal C5a peptidase is a highly specific endopeptidase. Infect. Immun. 60: 5219-5223.

Commons R., S. Rogers, T. Gooding, M. Danchin, J. Carapetis, R. Robins-Browne and N. Curtis. 2008. Superantigen genes in group A streptococcal isolates and their relationship with emm types. J. Med. Microbiol. 57: 1238-1246.

Courtney H.S., D.L. Hasty and J.B. Dale. 2002. Molecular mechanisms of adhesion, colonization, and invasion of group A streptococci. Ann. Med. 34: 77-87.

Cunningham M.W. 2000. Pathogenesis of group A streptococcal infections. Clin. Microbiol. Rev. 13: 470-511.

Edwards R.J., G.W. Taylor, M. Ferguson, S. Murray, N. Rendell, A. Wrigley, Z. Bai, J. Boyle, S.J. Finney, A. Jones, et al. 2005. Specific C-terminal cleavage and inactivation of interleukin- 8 by invasive disease isolates of Streptococcus pyogenes. J. Infect. Dis. 192: 783-790.

Enright M.C., B.G. Spratt, A. Kalia., J.H. Cross and D.E. Bessen. 2001. Multilocus sequence typing of Streptococcus pyogenes and the relationships between emm type and clone. Infect. Immun. 69: 2416-2427.

Facklam R., B. Beall, A. Efstratiou, V. Fischetti, D. Johnson, E. Kaplan, P. Kriz, M. Lovgren, D. Martin, B. Schwartz, et al. 1999. Emm typing and validation of provisional M types for group A streptococci. Emerg. Infect. Dis. 5: 247-253.

Ferretti J.J., W.M. McShan, D. Ajdic, D.J. Savic, G. Savic, K. Lyon, C. Primeaux, S. Sezate, A.N. Suvorov, S. Kenton, et al. 2001. Complete genome sequence of an M1 strain of Streptococcus pyogenes. Proc. Natl. Acad. Sci. USA 98: 4658-4663.

Fraser J., V. Arcus, P. Kong, E. Baker and T. Proft. 2000. Superantigens - powerful modifiers of the immune system. Mol. Med. Today 6: 125-132.

Green N.M., S. Zhang, S.F. Porcella, M.J. Nagiec, K.D. Barbian, S.B. Beres, R.B. LeFebvre and J.M. Musser. 2005. Genome sequence of a serotype M28 strain of group a streptococcus: potential new insights into puerperal sepsis and bacterial disease specificity. J. Infect. Dis. 192: 760-770.

Hartas J., M. Hibble and K.S. Sriprakash. 1998. Simplification of a locus-specific DNA typing method (Vir typing) for Streptococcus pyogenes. J. Clin. Microbiol. 36: 1428-1429.
Hauser A.R., D.L. Stevens, E.L. Kaplan and P.M. Schlievert. 1991. Molecular analysis of pyrogenic exotoxins from Streptococcus pyogenes isolates associated with toxic shock-like syndrome. J. Clin. Microbiol. 29: 1562-1567.

Herschleb J., G. Ananiev and D.C. Schwartz. 2007. Pulsed-field gel electrophoresis. Nat. Protoc. 2: 677-684.

Hoe N., K. Nakashima, D. Grigsby, X. Pan, S.J. Dou, S. Naidich, M. Garcia, E. Kahn, D. Bergmire-Sweat and J.M. Musser. 1999. Rapid molecular genetic subtyping of serotype M1 group A Streptococcus strains. Emerg. Infect. Dis. 5: 254-263.

Koller T., A.G. Manetti, B. Kreikemeyer, C. Lembke, I. Margarit, G. Grandi and A. Podbielski. 2010. Typing of the pilus-proteinencoding FCT region and biofilm formation as novel parameters in epidemiological investigations of Streptococcus pyogenes isolates from various infection sites. J. Med. Microbiol. 59: 442-452.

Kurupati P., C.E. Turner, I. Tziona, R.A. Lawrenson, F.M. Alam, M. Nohadani, G.W. Stamp, A.S. Zinkernagel, V. Nizet, R.J. Edward, et al. 2010. Chemokine-cleaving Streptococcus pyogenes protease SpyCEP is necessary and sufficient for bacterial dissemination within soft tissues and the respiratory tract. Mol. Microbiol. 76: 1387-1397.

Lintges M., S. Arlt, P. Uciechowski, B. Plumakers, R.R. Reinert, A. Al-Lahham, R. Lutticken and L. Rink. 2007. A new closed-tube multiplex real-time PCR to detect eleven superantigens of Streptococcus pyogenes identifies a strain without superantigen activity. Int. J. Med. Microbiol. 297: 471-478.

Matsumoto M., N.P. Hoe, M. Liu, S.B. Beres, G.L. Sylva, C.M. Brandt, G. Haase and J.M. Musser. 2003. Intrahost sequence variation in the streptococcal inhibitor of complement gene in patients with human pharyngitis. J. Infect. Dis. 187: 604-612.

Maxted W.R., J.P. Widdowson, C.A. Fraser, L.C. Ball and D.C. Bassett. 1973. The use of the serum opacity reaction in the typing of group-A streptococci. J. Med. Microbiol. 6: 83-90.

Moody M.D., J. Padula, D. Lizana and C.T. Hall. 1965. Epidemiologic Characterization of Group A streptococci by T-Agglutination and M-Precipitation Tests in the Public Health Laboratory. Health. Lab. Sci. 2: 149-162.

Musser J.M., A.R. Hauser, M.H. Kim, P.M. Schlievert, K. Nelson and R.K. Selander. 1991. Streptococcus pyogenes causing toxicshock-like syndrome and other invasive diseases: clonal diversity and pyrogenic exotoxin expression. Proc. Natl. Acad. Sci. USA 88: 2668-2672.

Nandi S., N.K. Ganguly, R. Kumar, D.K. Bakshi, V.S. Vivek Sagar and A. Chakraborti. 2008. Genotyping of group A Streptococcus by various molecular methods. Indian. J. Med. Res. 127: 71-77.

Oehmcke S., O. Shannon, M. Morgelin and H. Herwald. 2010. Streptococcal M proteins and their role as virulence determinants. Clin. Chim. Acta 411: 1172-1180.

Perez-Caballero D., I. Garcia-Laorden, G. Cortes, M.R. Wessels, S.R. de Cordoba and S. Alberti. 2004. Interaction between complement regulators and Streptococcus pyogenes: binding of C4b-binding protein and factor $\mathrm{H} /$ factor $\mathrm{H}$-like protein 1 to M18 strains involves two different cell surface molecules. J. Immunol. 173: 6899-6904

Pinto F.R., J. Melo-Cristino and M. Ramirez. 2008. A confidence interval for the wallace coefficient of concordance and its application to microbial typing methods. PLoS One. 3: e3696.

Proft T., S.L. Moffatt, K.D. Weller, A. Paterson, D. Martin and J.D. Fraser. 2000. The streptococcal superantigen SMEZ exhibits wide allelic variation, mosaic structure, and significant antigenic variation. J. Exp. Med. 191: 1765-1776.

Richardson L.J., S.Y. Tong, R.J. Towers, F. Huygens, K. McGregor, P.K. Fagan, B.J. Currie, J.R. Carapetis and P.M. Giffard. 2010. Preliminary validation of a novel high resolution melt-based typing method based on the multilocus sequence typing scheme of Streptococcus pyogenes. Clin. Microbiol. Infect. in press. 
Schmitz F.J., A. Beyer, E. Charpentier, B.H. Normark, M. Schade, A.C. Fluit, D. Hafner and R. Novak. 2003. Toxin-gene profile heterogeneity among endemic invasive European group A streptococcal isolates. J. Infect. Dis. 188: 1578-1586.

Seppala H., J. Vuopio-Varkila, M. Osterblad, M. Jahkola, M. Rummukainen, S.E. Holm and P. Huovinen. 1994. Evaluation of methods for epidemiologic typing of group A streptococci. J. Infect. Dis. 169: 519-525.

Slater G.W. 2009. DNA gel electrophoresis: the reptation model(s). Electrophoresis 30 Suppl. 1: S181-187.

Smeesters P. R., D.J. McMillan and K.S. Sriprakash. 2010. The streptococcal M protein: a highly versatile molecule. Trends. Microbiol. 18: 275-282.

Stanley J., D. Linton, M. Desai, A. Efstratiou and R. George. 1995. Molecular subtyping of prevalent M serotypes of Streptococcus pyogenes causing invasive disease. J. Clin. Microbiol. 33: 2850-2855.

Stevens D.L., M.H. Tanner, J. Winship, R. Swarts, K.M. Ries, P.M. Schlievert and E. Kaplan. 1989. Severe group A streptococcal infections associated with a toxic shock-like syndrome and scarlet fever toxin A. N. Engl. J. Med. 321: 1-7

Sumby P., K.D. Barbian, D.J. Gardner, A.R. Whitney, D.M. Welty, R.D. Long, J.R. Bailey, M.J. Parnell, N.P. Hoe, G.G. Adams, et al. 2005. Extracellular deoxyribonuclease made by group A Streptococcus assists pathogenesis by enhancing evasion of the innate immune response. Proc. Natl. Acad. Sci. USA 102: 1679-1684.

Sumby P., S. Zhang, A.R. Whitney, F. Falugi, G. Grandi, E.A. Graviss, F.R. Deleo and J.M. Musser. 2008. A chemokinedegrading extracellular protease made by group A Streptococcus alters pathogenesis by enhancing evasion of the innate immune response. Infect. Immun. 76: 978-985.

Swift H.F., A.T. Wilson and R.C. Lancefield. 1943. Typing Group A Hemolytic streptococci by $\mathrm{M}$ precipitin reactions in capillary pipettes. J. Exp. Med. 78: 127-133.

Szczypa K., E. Sadowy, R. Izdebski and W. Hryniewicz. 2004. A rapid increase in macrolide resistance in Streptococcus pyogenes isolated in Poland during 1996-2002. J. Antimicrob. Chemother. 54: 828-831.

Tart A.H., M.J. Walker and J.M. Musser. 2007. New understanding of the group A Streptococcus pathogenesis cycle. Trends. Microbiol. 15: 318-325.

Tenover F.C., R.D. Arbeit, R.V. Goering, P.A. Mickelsen, B.E. Murray, D.H. Persing and B. Swaminathan. 1995. Interpreting chromosomal DNA restriction patterns produced by pulsedfield gel electrophoresis: criteria for bacterial strain typing. J. Clin. Microbiol. 33: 2233-2239.

Terao Y., Y. Mori, M. Yamaguchi, Y. Shimizu, K. Ooe, S. Hamada and S. Kawabata. 2008. Group A streptococcal cysteine protease degrades C3 (C3b) and contributes to evasion of innate immunity. J. Biol. Chem. 283: 6253-6260.

Unnikrishnan M., D.M. Altmann, T. Proft, F. Wahid, J. Cohen, J.D. Fraser and S. Sriskandan. 2002. The bacterial superantigen streptococcal mitogenic exotoxin $\mathrm{Z}$ is the major immunoactive agent of Streptococcus pyogenes. J. Immunol. 169: 2561-2569.

von U. Pawel-Rammingen, B.P. Johansson and L. Bjorck. 2002. IdeS, a novel streptococcal cysteine proteinase with unique specificity for immunoglobulin G. Embo. J. 21: 1607-1615.

Walker M.J., A. Hollands, M.L. Sanderson-Smith, J.N. Cole, J.K. Kirk, A. Henningham, J.D. McArthur, K. Dinkla, R.K. Aziz, R.G. Kansal, et al. 2007. DNase Sda1 provides selection pressure for a switch to invasive group A streptococcal infection. Nat. Med. 13: 981-985.

C.E. Yu and J.J. Ferretti. 1989. Molecular epidemiologic analysis of the type A streptococcal exotoxin (erythrogenic toxin) gene (speA) in clinical Streptococcus pyogenes strains. Infect. Immun. 57: 3715-3719.

Zinkernagel A.S., A.M. Timmer, M.A. Pence, J.B. Locke, J.T. Buchanan, C.E. Turner, I. Mishalian, S. Sriskandan, E. Hanski, and V. Nizet. 2008. The IL-8 protease SpyCEP/ScpC of group A Streptococcus promotes resistance to neutrophil killing. Cell. Host. Microbe. 4: 170-178. 